\title{
Design and Implementation of a Low-Power Energy Management Module with Emergency Reserve for Solar Powered DTN-Nodes
}

\author{
Michael Doering, Stephan Rottmann, Lars Wolf \\ Institute of Operating Systems and Computer Networks \\ Technische Universität Braunschweig \\ Braunschweig, Germany \\ (mdoering|rottmann|wolf)@ibr.cs.tu-bs.de
}

\begin{abstract}
DTN nodes often idly wait for contacts and unnecessarily consume energy during these periods. For solar-powered nodes this means that solar panels and batteries could be much smaller with an efficient energy management, reducing the physical size of these nodes. We present our design of a solar-powered DTN node including a module that handles solar charge management, energy management and a discovery mechanism to wake sleeping nodes. Moreover, the system senses the remaining battery capacity, and keeps a reserve for emergency communications. Our evaluation shows that the power management module's design is energy-efficient and performs as intended. Furthermore, we evaluate the discovery mechanism. The results show that the capabilities of the module offer a promising new approach to the implementation of energy efficient routing in DTNs.
\end{abstract}

\section{Keywords}

Delay Tolerant Networks, Solar Power, Energy Harvesting, Extreme Communication

\section{INTRODUCTION}

Photovoltaic cells are a good option for powering DTNnodes in remote areas which do not have sufficient infrastructure support. However, bundle protocol agents require non-negligible processing power and most DTN-nodes usually require a high bandwidth long range wireless interface. This results in a typical power consumption in the order of a few Watts and requires a certain area of solar panel and a certain rechargeable battery capacity. In other words: solar-powered DTN-nodes with a decent performance are large and bulky.

In order to reduce the physical dimensions it is necessary to reduce the power consumption by implementing power management. A common approach is to power-off unneeded components and to reduce the chip frequency of underutilized resources.

Permission to make digital or hard copies of all or part of this work for personal or classroom use is granted without fee provided that copies are not made or distributed for profit or commercial advantage and that copies bear this notice and the full citation on the first page. To copy otherwise, to republish, to post on servers or to redistribute to lists, requires prior specific permission and/or a fee.

ExtremeCom2011, September 26-30, 2011, Manaus, Brazil.

Copyright 2011 ACM XXX-X-XXXX-XXXX-X/11/09 ...\$10.00
Our approach goes a step further: we propose a dedicated power management module (PMM) to switch the complete DTN-node off. Only the PMM remains active (but in a very deep sleep mode) and wakes the node e.g., on contact with another specific node. The PMM also features a low-power IEEE 802.4.15 radio for discovery and to communicate with other nodes' PMMs in order to negotiate complex wakeup rules, e.g., 'wake only if battery is above $30 \%$ or if bundle is urgent'. Moreover, the PMM handles battery charging and accounting, and keeps an emergency reserve for urgent communication.

In this paper, we present our solar-powered DTN-node, the PMM hardware design and its firmware functions. Besides the obvious advantages of power management, our system is also beneficial for an easy implementation of energy-aware opportunistic routing, since a DTN-router can upload dynamic wakeup-rules to the PMM before the node is powered off. Our vision is a DTN in which the majority of nodes are turned off while their batteries are charging, instead of being drained by a node that idly waits for a contact.

Our approach is similar to the dual-radio two-tier architecture presented in [1], but there are several significant differences. First, we use IEEE 802.15.4 for discovering other nodes instead of a proprietary radio. This standardized wireless technology makes our design more inter-operable since there is a variety of radios available (e.g. USB dongles) and it can be expected that 802.15 .4 will be integrated in future smartphones and laptops. Moreover, the power consumption in receive mode is lower, making discovery in 'deep sleep' mode more energy efficient. Second, instead of using mobility prediction, our approach uses selective discovery based on DTN routing decisions, i.e., the PMMs actively negotiate wakeup events based on routing and remaining battery capacity. This allows to reserve a certain capacity for urgent or emergency communications. And third, our DTN-node is deliberately designed to be as simple and robust as possible. In that regard our design is similar to the solar-powered

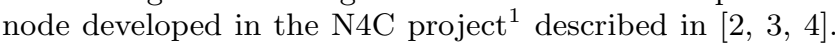
We also decided for sealed lead acid batteries, because these a more robust and safe than LiPo-batteries, although the energy-density is lower. However, lead acid batteries are much easier to obtain and available even in very remote areas - an important requirement in developing regions. But besides using solar cells and the same type of batteries to power a DTN node, our design is very different from the N4C

\footnotetext{
${ }^{1}$ http://www.n4c.eu/
} 
design. Our PMM handles energy management and does not require an additional charge controller. Moreover, to the best of our knowledge, the N4C design does not implement a sleep mode and has no second radio for discovery.

The remainder of this paper is structured as follows. In Section 2 the application scenarios that motivated the development of the PMM are introduced. Section 3 first gives an overview of the system design and then continues with a detailed description of the hardware and software components. In Section 4 we present the evaluation of the solar charge management, discovery range and energy consumption. Finally, we conclude the paper.

\section{APPLICATION SCENARIOS}

Statinary DTN-nodes are useful in various scenarios. The main application area of solar-powered nodes are remote locations without mains infrastructure, but also in other cases, even in urban settings, such a self-contained DTNnode can be useful due to its simplicity and fast installation. Usually (but not necessarily) these nodes are stationary and located at elevated positions for better radio coverage. The following exemplary application scenarios are common for such systems:

\subsection{Bundle Relay for Message Ferries}

In this scenario the solar-powered DTN node's task is mainly to store and forward messages from and to ferries. This means that the node is positioned at the path of regularly passing ferries, and spends much time idly waiting for a contact. Here the PMM comes into play and wakes the node shortly before a contact. For deterministic contacts the PMM can use a schedule (time triggered wakeup) and does not require the low power radio, but for non-deterministic contacts, a low power 802.15.4 radio on the ferry sends beacons to the PMM (selective discovery triggered wakeup). Upgrading an existing ferry node with such a radio should be unproblematic, since there are several inexpensive and energy-efficient 802.15.4 transceivers available (e.g. 'USBdongles'). Of course, we recommend using a PMM for new designs of ferry nodes.

\subsection{DTN Access for Mobile Users}

In this scenario, the solar-powered DTN node provides DTN access to mobile users who only sporadically use the network, e.g. for sending and receiving text or voice messages. Again, the solar-powered node is mainly idle. If a user needs service a wakeup signal is sent from the user's mobile device to the PMM. Currently almost all smartphones are equipped with Bluetooth, and the PMMs modular hardware also allows to use Bluetooth for discovery. However, we strongly favor 802.15.4 for its better energy-efficiency, range and security, and we expect that future smartphones will include 802.15.4 radios. Moreover, laptops are easily upgraded with such interfaces. As a last resort there is a port pin on the PMM to externally trigger a wakeup, e.g., by a motion sensor or simply a push button.

\subsection{Delay-tolerant on Demand Backbone}

In this scenario, several solar-powered, fixed DTN nodes operate as backbone between remote locations. Differently from the usual DTN assumption, there is a stable wireless link between the nodes. However, we assume that there are energy constraints so that there is only an intermittent power supply available. This is a very reasonable assumption for solar-powered nodes, because even with highly oversized (and therefore very expensive) solar panels and batteries it is hardy possible to guarantee continuous operation in all weather conditions and all seasons.

All backbone nodes are equipped with a PMM, which is used to wake up the next hop node. In this scenario there are several battery capacity thresholds. First, if the battery is almost full and the sun is shining a node is always on, to ensure a low latency and because there is no reason to switch the node off while the solar cells harvest enough energy. Second, if the battery is neither almost full nor almost empty, the node is powered off if there are no bundles to route; and selectively powered on via the PMM if there is work to do. It can be expected that nodes spend most of their time in this "on demand" mode. Third, if the batteries of the nodes are below the emergency energy budget, the nodes stay powered off unless high priority bundles are transmitted. To distinguish between the different levels of urgency of bundles, the two bit priority field according to RFC5050 can be used.

\section{SYSTEM DESIGN}

The block diagram in figure 1 shows two possible DTN node configurations. On the left is a DTN node equipped with an PMM, which is capable to switch on/off the embedded system running Linux and a DTN daemon. The PMM triggers a wakeup event if it receives a wakeup message on its integrated IEEE 802.15.4 radio. This beacon is either transmitted from another PMM, or by another DTN node without a PMM. In this case the node requires an 802.15.4 interface, as shown on the right.

This paper focuses on the power management module (PMM), but a realistic evaluation of the PMM is only possible with a solar-powered DTN node. For this reason we also present our node in section 3.2.

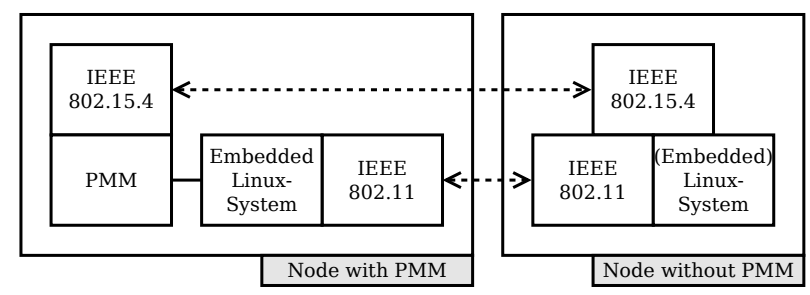

Figure 1: Possible DTN node configurations

\subsection{Power Management Module}

The PMM's architecture shown in figure 2 is based on an Atmega644P MCU, which connects via an FT232RL serialto-USB chip with the DTN node. Moreover, the PMM has a socket for low power radios, which is currently populated with an XBee-Pro[5] 802.15.4 radio, as shown in figure 4. However, there are several other low power radios available with different PHY/MAC technologies but with the same footprint and pin-out. This makes our design very versatile, and allows compliance with (and exploitation of) different national radio spectrum regulations. As an additional feature the PMM can enable a virtual serial port (see right side in figure 2) that allows the DTN-node direct access to the low power radio, to use it as if it were its own wireless interface. 
The MCU also controls the MOSFET switches used for battery charging via the solar panel, and for switching the DTN node's power supply. Current sensing is implemented with INA138 integrated circuits. The voltages are measured using potential dividers which are duty-cycled to preserve energy. The remaining battery capacity is determined based on the current and voltage data over time. If it falls below a threshold value the DTN node is switched off, to preserve an energy budget for emergency communications. It is switched on again if the PMM's low power radio receives a message with an emergency flag; and also if the battery is charged above the threshold value. A DS1629 real time clock (RTC) provides persistent time, so that the MCU can go to sleep mode. As an additional feature the DS1629 contains a temperature sensor, which is helpful to ensure that the battery is not charged if its temperature specifications are exceeded. All components are mounted to a single printed circuit board. The photo in figure 3 shows the PMM's topside.

Although the PMM was primarily designed for solar-powered DTN nodes, it is notable that other unconventional power sources like wind wheels or muscle powered generators also can be used. It even may make sense to use the PMM with a conventional power supply and without a battery, in order to benefit from the energy-saving discovery and wakeup features.

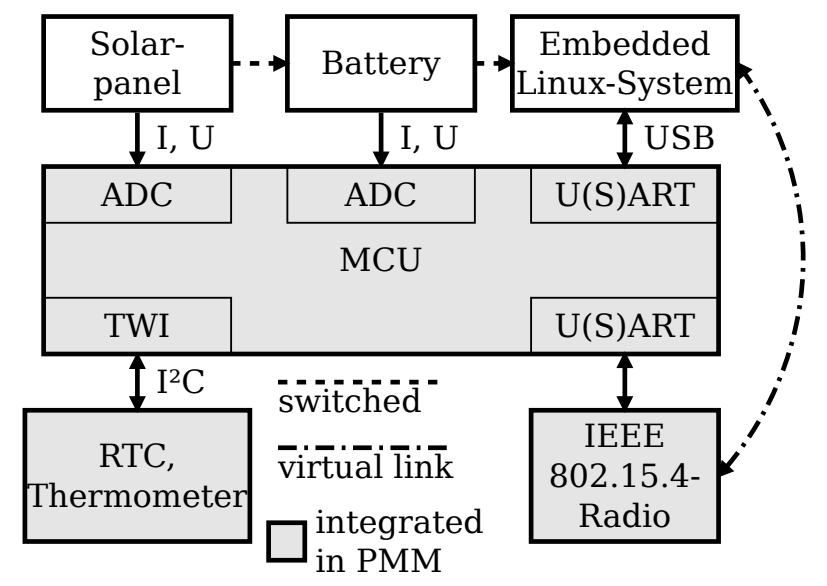

Figure 2: Architecture of the PMM

\subsubsection{Radio Control Protocol}

This section gives an overview of the currently implemented PMM control messages on the 802.15 .4 channel. It is very likely that additional messages will be implemented, since the PMM allows for a wide range of applications, which may benefit from future protocol extensions.

WHOISTHERE_REQUEST and _RESPONSE A mobile node sends this request as a broadcast message in order to discover other stations. As a parameter, its DTN name is transmitted. An answering node sends its name, geographic position and remaining battery capacity.

STATUS_REQUEST and _RESPONSE Queries a known node to send detailed information on voltages, currents etc.

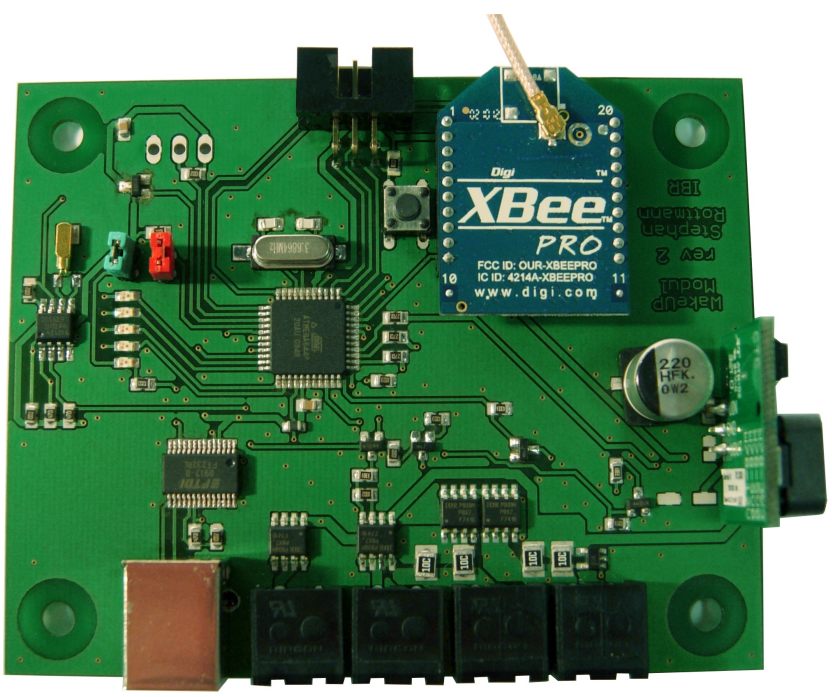

Figure 3: Top side of the PMM

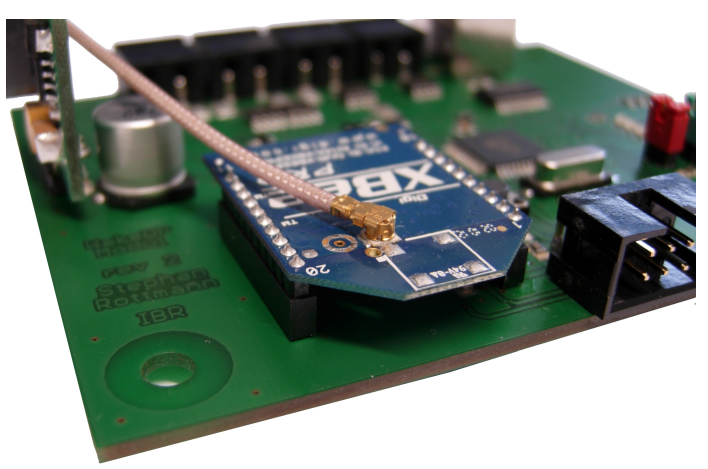

Figure 4: 802.15.4 XBee radio plugged into the radio-socket of the $\mathrm{PMM}$

WAKEUP_REQUEST and_RESPONSE This message schedules a wakeup event at a remote PMM. It contains the name of the DTN-node, time for the scheduled wakeup, wakeup duration and a priority. The remote PMM uses the priority and its remaining battery capacity to decide if the request is acknowledged.

GETNEXTEVENT_REQUEST and _RESPONSE A mobile node uses this command to find out when (and if) the DTN-system is powered up the next time.

BEACON A node may announce its presence by periodically transmitting a short beacon with its name. This message provides an alternative to the WHOISTHERE query.

\subsubsection{PMM Control Daemon}

The PMM and the control daemon running on the embedded Linux system communicate via an USB interface. Applications can connect via TCP sockets to the daemon, and request information on the current energy state. Moreover, registered applications are notified before the node is powered off. The daemon also handles energy monitoring and PMM configuration. This includes uploading scheduled 
wakeup events and energy thresholds to the PMMs nonvolatile memory. Furthermore, the daemon is able to provide a virtual serial port that allows direct access to the serial port of the low power radio module. This feature is useful for our future low power radio convergence layer, which allows the DTN node to send small bundles more energy efficient.

\subsubsection{Wakeup Strategies}

In our current version there are three strategies implemented to wake up the DTN node.

By Request Another station can send a request including start, duration, and priority to the PMM, to wake up the DTN node via the 802.15.4-connection. With help of the transmitted priority, the module can decide if there is sufficient energy stored in the battery or if enough energy can be harvested by the PV-panel. If the amount is to small at the beginning of the requested time span, the event will not be discarded, but the DTN board will not be powered on either. This provides an opportunity to boot the node later, if the energy collected by the node in the meantime exceeds the limit needed for the requested priority.

By Contact The DTN node can configure the PMM to trigger a wakeup if the PMM receives a beacon from a specific node. Then the node issues a shutdown request to the PMM and sleeps until the specific node is within range or another wakeup event occurs.

Opportunistic For a system that should be able to work reliable all over the year, the components to collect and store electrical energy will probably be - depending on the region - oversized for summer seasons. Since the battery can store only a limited amount of energy, it will likely come to a point at which it can happen that the battery is fully charged but there is still energy provided by the solar panel. To use it, the DTN node will be powered up even if it was not requested.

\subsection{Solar-Powered DTN node}

The solar-powered DTN node was developed in the Optracom project ${ }^{2}$. For this reason the DTN node hardware is based on the Optracom node design. A Ubiquiti RouterStation (an embedded router platform) runs the OpenWRT embedded Linux distribution. It provides several miniPCI sockets and USB ports for extension. An UB5 802.11a interface card is installed and connected to an external antenna with $10 \mathrm{dbi}$ gain. The node runs the IBR-DTN[6] bundle protocol agent. Figure 5 shows the basic setup. Both the solar panel and the battery connect to the PMM, which manages charging and also switches the RouterStations power. The RouterStation connects via USB to the PMM.

The photo in figure 6 shows the internals of the node's case. The battery is at the botton, the PMM on the upper left, besides the RouterStation. Also note the vertical, internal PMM antenna. The complete system with external antenna and solar panel is shown in figure 10 .

\subsection{Mobile Nodes}

As stated earlier, solar-powered nodes are commonly stationary. However, several use cases require mobile nodes (mainly mobile users and ferries) to send wakeup-requests

\footnotetext{
${ }^{2}$ http://www.optracom.de/
}

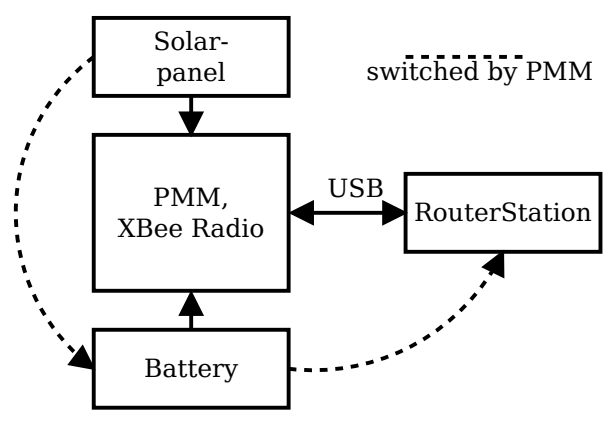

Figure 5: Simplified block diagram of the system

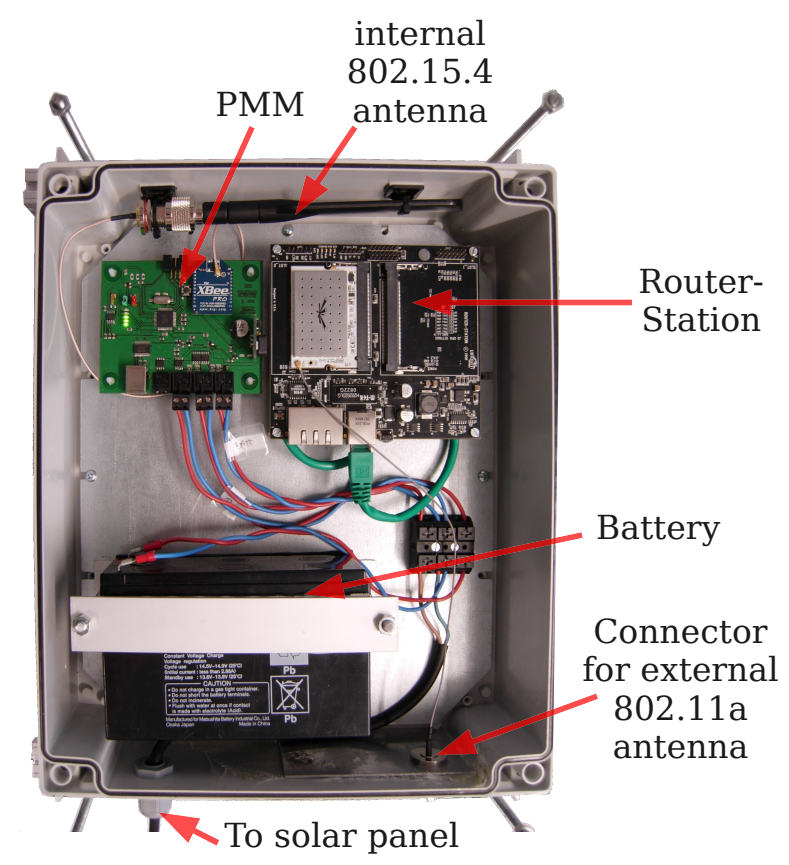

Figure 6: The complete system with opened case

to the PMM by an 802.15.4 interface. 802.15.4. is widely used in wireless sensor networks and industrial controls, but not yet integrated into regular smartphones. For evaluation purposes we used an USB-stick[7], shown in figure 7. Figure 8 shows a mobile node for vehicles operating as message ferries. It is equipped with several dual- and tri-band antennas and will be used in a future evaluation of different low power radios.

\section{EVALUATION}

In this section we present our preliminary evaluation results. The evaluation setup is shown in figure 10. The graph in figure 9 shows the line of sight range of the 802.15.4 and the $802.11 \mathrm{a}$ radios. Note that 802.15 .4 operates with a transmit power of $18 \mathrm{dBm}$ on an internal vertical $3 \mathrm{dbi}$ gain antenna while $802.11 \mathrm{a}$ operates with $23 \mathrm{dBm}$ on an external $10 \mathrm{dbi}$ antenna. However, the 802.15.4 range still achieves a higher range. Therefore, a wakeup can be requested by a mobile node before it is within $802.11 \mathrm{a}$ range. This is important to maximize contact time, because the DTN node needs some seconds to boot up. 


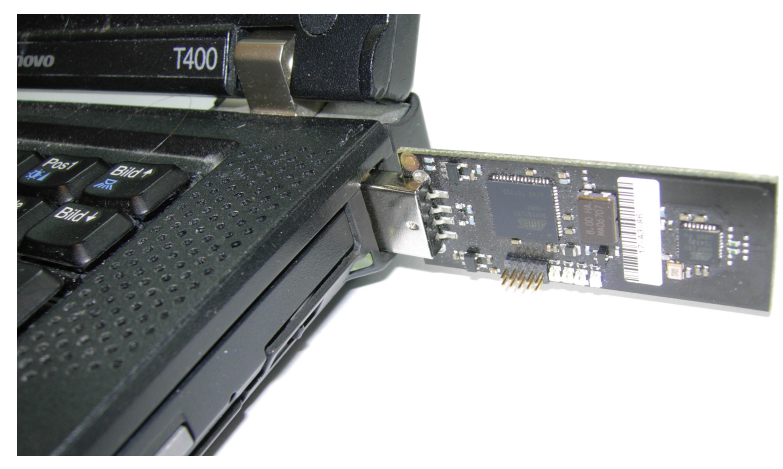

Figure 7: An USB-stick with IEEE 802.15.4 functionality by Atmel

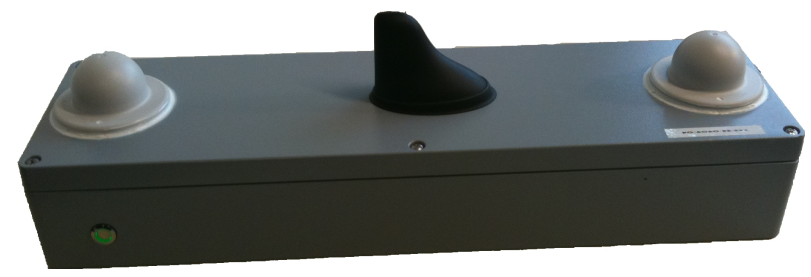

Figure 8: Node for message ferries

Figure 11 shows a graph of the voltages and currents in the system. On the left side of the graph the sun is shining and the harvested energy exceeds the needed energy. Therefore, the battery is charged. Since the battery is almost full, the charging process is interrupted frequently as the battery voltage reaches the upper threshold. On the right side of the graph it gets more cloudy. The amount of harvested energy is now below the consumed energy, and the difference is discharged from the battery. Around 850 seconds the battery is charged again for a short period, but does not reach the upper threshold.

The following table shows the energy consumption of the main components. The PMMs MCU consumes roughly $13 \mathrm{~mW}$, but most energy is used by the XBee radio - more than $450 \mathrm{~mW}$ for constant operation. But we assume a rea-

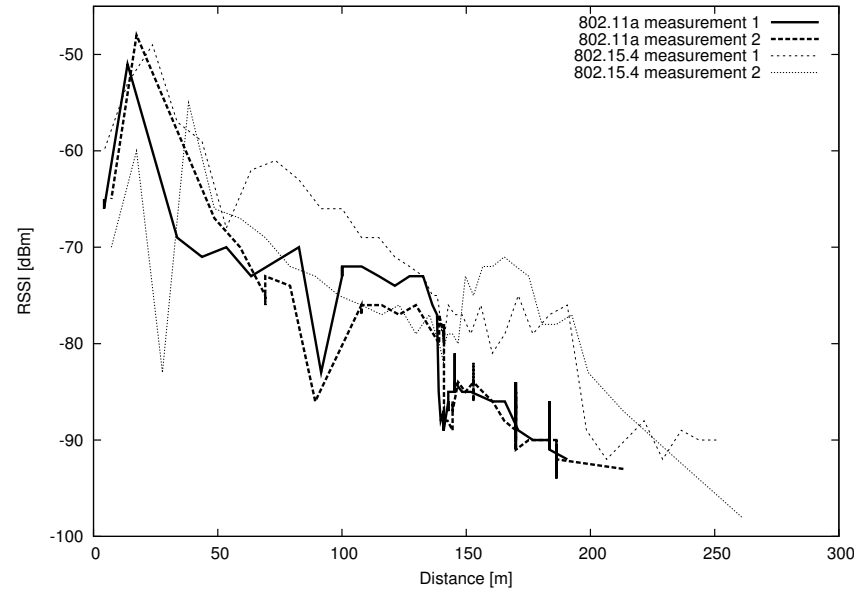

Figure 9: Distance-vs-RSSI from 802.11a and 802.15.4 radios

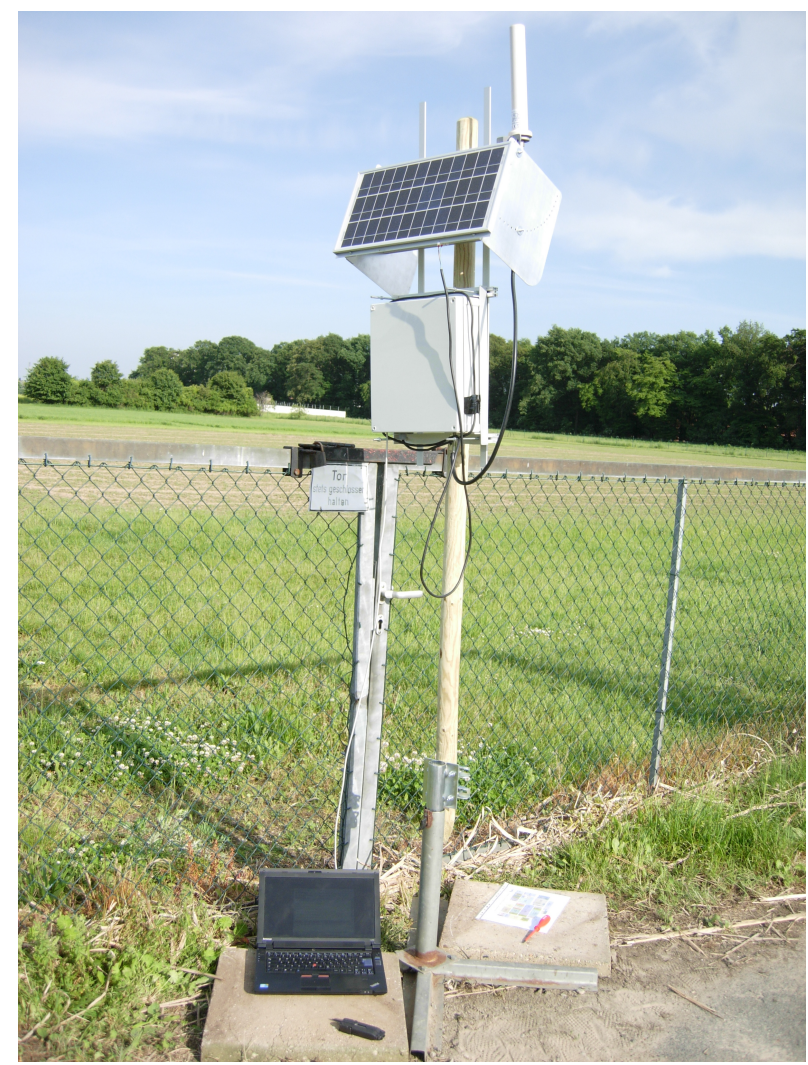

Figure 10: Evaluation setup

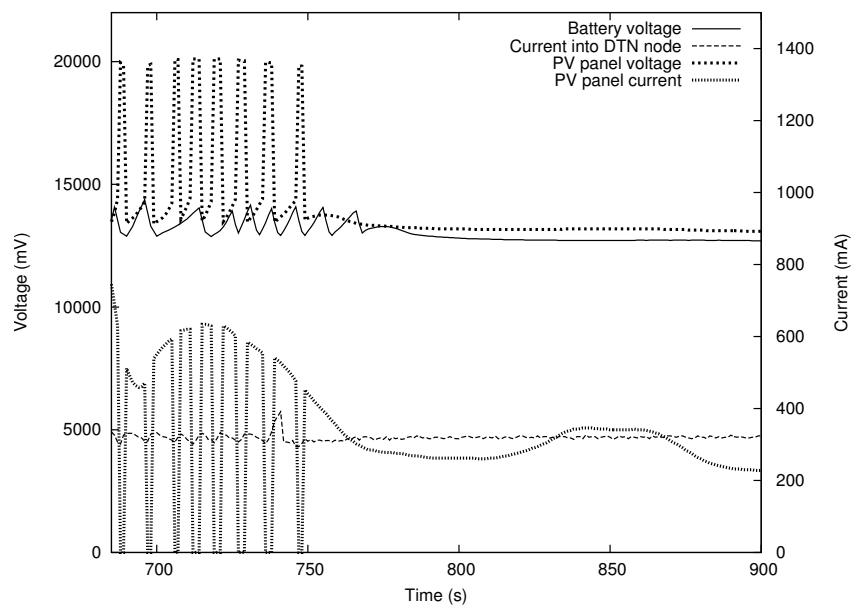

Figure 11: Voltages and Currents during charging and discharging of the battery 
sonable duty cycle of only $15 \%$ is sufficient for an acceptable discovery latency. Another $36 \mathrm{~mW}$ are dissipated by the voltage regulator. In total the PMM consumes less than $117 \mathrm{~mW}$. The embedded Linux-system adds an amount of $5000 \mathrm{~mW}$ average when it is running.

\begin{tabular}{l|cc|c}
\hline Component & I [mA] & P [mW] & Duty Cycle \\
\hline \hline $\mathrm{uC}$ & 3 & 12.375 & $100 \%$ \\
XBee & 110 & 453.75 & $15 \%$ \\
\hline Voltage Reg. & 3 & 36 & $100 \%$ \\
\hline Total & \multicolumn{3}{|c}{$116.4375 \mathrm{~mW}$} \\
\hline
\end{tabular}

\section{CONCLUSIONS AND FUTURE WORK}

The combination of a solar charging circuit with a low power radio for discovery and remote wakup mechanisms into a single module benefits from synergies, e.g. only a single MCU and a single voltage regulator is required. Therefore, our design has lower physical dimensions, costs, and a lower energy consumption than seperate modules for charging, swiching, metering and discovery.

The range measurements have shown that the range of the discovery radio exceeds $802.11 \mathrm{a}$ and therefore our design works as intended. For mobile scenarios a sleeping node can be booted up before the 802.11 a contact starts. For future work we plan the evaluation of several other discovery radios, especially since most of the PMM's energy consumption is caused by the radio. To extend the range of the 802.15.4 radio, an external antenna can be used. Another possible approach is to implement scheduled deep sleep modes with a very low duty cycle, in which the radio listens only every few minutes for a wakup signal. However, this would increase the latency and might therefore not be acceptable for some applications.

Another area of future work is the development of an interface between the PMM control daemon and the DTN bundle protocol agent. This allows for energy aware DTN routing and the integration of bundle priorities into the wakeup strategies. Moreover, since the DTN node can direcly access the PMM's radio, a low bandwidth convergence layer could be implemented that uses the PMM's radio. Another interesting approach is that the PMM could receive and buffer bundles while the DTN-node sleeps or is in the process of booting up.

\section{ACKNOWLEDGEMENTS}

This work was supported in part by the European Regional Development Fund under project number W2-80028895.

\section{REFERENCES}

[1] N. Banerjee, M. D. Corner, and B. N. Levine, "Design and Field Experimentation of an Energy-Efficient Architecture for DTN Throwboxes," IEEE/ACM Transactions on Networking, vol. 18, no. 2, pp. 554-567, April 2010.

[2] E. Meehan and K. Hartnett, "N4c dtn node user documentation, dtn node build," Report, 32011. [Online]. Available: http://www.n4c.eu/Download/ n4c-wp5-052-dtn-node-build-12\%5B1\%5D.pdf

[3] S. Farrell, "N4c dtn node design," Report, 52009. [Online]. Available: http: //www.n4c.eu/Download/n4c-tcd-006-d5_1-FINAL.pdf

[4] S. Farrell, S. Weber, A. McMahon, E. Meehan, and K. Hartnett, "An n4c dtn router node design," in 1st Extreme Workshop on Communication, Laponia, Sweden, August 8-14 2009., aug 2009.

[5] Digi International Inc., "XBee/XBee-PRO RF Modules," Datasheet, Minnetonka, USA, Sep. 2009. [Online]. Available: http: //ftp1.digi.com/support/documentation/90000982_B.pdf

[6] M. Doering, S. Lahde, J. Morgenroth, and L. Wolf, "Ibr-dtn: an efficient implementation for embedded systems," in Proceedings of the third ACM workshop on Challenged networks, ser. CHANTS '08. New York, NY, USA: ACM, 2008, pp. 117-120. [Online]. Available: http://doi.acm.org/10.1145/1409985.1410008

[7] Atmel Corporation, "AVR2016: RZRAVEN Hardware User's Guide," Application Note, San Jose, USA, Apr. 2008. [Online]. Available: http://www.atmel.com/dyn/ resources/prod_documents/doc8117.pdf 